\title{
Clinical Utility of Guselkumab in the Treatment of Moderate-to-Severe Plaque Psoriasis
}

This article was published in the following Dove Press journal:

Clinical, Cosmetic and Investigational Dermatology

\author{
Jeremy G Light $\mathbb{D}^{\prime}$ \\ Jennifer J Su' \\ Steven R Feldman (1D ${ }^{1-4}$ \\ 'Center for Dermatology Research, \\ Department of Dermatology, Wake \\ Forest School of Medicine, Winston- \\ Salem, NC, USA; ${ }^{2}$ Department of \\ Pathology, Wake Forest School of \\ Medicine, Winston-Salem, NC, USA; \\ ${ }^{3}$ Department of Social Sciences \& Health \\ Policy, Wake Forest School of Medicine, \\ Winston-Salem, NC, USA; ${ }^{4}$ Department \\ of Dermatology, University of Southern \\ Denmark, Odense, Denmark
}

\begin{abstract}
Psoriasis is a chronic immune-mediated disease involving complex interaction of $\mathrm{T}$ cells and keratinocytes. The comprehensive pathogenesis of psoriasis is not fully understood but the IL-23/Th17 axis is a central pathway in driving disease development. Guselkumab is the first treatment of moderate-to-severe psoriasis that specifically targets the p19 subunit of IL-23. The benefit of guselkumab has been established by a number of clinical trials including demonstration of greater long-term efficacy in recent comparator trials. This review addresses the results of head-to-head trials (ECLIPSE, IXORA-R, and POLARIS) that compared guselkumab to secukinumab, ixekizumab, and fumaric acid esters. The previously demonstrated long-term efficacy of guselkumab has been corroborated by many recently published studies. The effective and safe profile, convenient dosing, and improved quality of life in patients make gulselkumab a viable first-line treatment option for moderate-to-severe psoriasis.
\end{abstract}

Keywords: guselkumab, psoriasis, biologics, efficacy, interleukin-23

\section{Introduction}

Psoriasis is a chronic immune disease that causes inflammatory skin lesions and affects over 125 million people. ${ }^{1-3}$ Psoriasis is characterized by systemic inflammation and resulting associations with serious comorbidity, including psoriatic arthritis, inflammatory bowel disease, cardiovascular disease, psychiatric diseases, and an increased risk for death. ${ }^{1-5}$ For those with moderate-to-severe disease, systemic biologic therapies can prevent disability and may reduce risk of comorbid disease. $^{6-9}$ Recent advances in disease understanding have led to the development of highly effective and targeted therapies for the treatment of moderate-to-severe psoriasis (and the development of highly effective treatments has advanced our understanding of the disease). ${ }^{10-13}$ The characterization of the critical role of interleukin (IL)-23 in the pathogenic pathway has resulted in a shift in therapeutic targeting for better psoriasis treatments. ${ }^{14}$

\section{IL-23 and Psoriasis Pathogenesis}

IL-23 is a heterodimeric cytokine composed of p19 and p40 subunits. IL-23 is a crucial component in the pathogenesis of psoriasis. ${ }^{15}$ The IL-23/Th17 axis is a central pathway in the development of the disease. ${ }^{14,16,17}$ IL-23 levels in the serum and skin lesions are increased in patients with psoriasis. ${ }^{18,19}$ Psoriasis is a multifactorial disease of complex immune activation in susceptible individuals. Proinflammatory cytokines - including IL-1 $\beta$, IL-6, and TNF- $\alpha$ - activate dermal dendritic cells causing increased production
Correspondence: Jeremy G Light Department of Dermatology, Wake Forest School of Medicine, Medical Center Boulevard, Winston-Salem, NC 27I57-107I, USA

Tel + I 336-716-7740

Fax + I336-716-7732

Email jlight2020@gmail.com 
of IL-23. ${ }^{15,16,20} \mathrm{IL}-23$ is the primary regulator for induction and maintenance of proinflammatory Th17 cell populations responsible for driving development of disease in psoriasis. ${ }^{14,21}$ The expansion of Th17 cells results in considerable amounts of IL-17 which stimulates a feedforward inflammatory response that causes epidermal hyperplasia, keratinocyte immune activation, and tissue inflammation. $^{14-16,21}$

The first approved biologic therapy to inhibit this pathway was ustekinumab, a fully human monoclonal antibody against the shared p40 subunit of the IL-12 and IL-23 cytokines, followed by the approval of several IL17 inhibitors. Although these treatments are effective, the subsequent discovery of IL-23 as the "master regulator" of Th17 cells led to the development of several antagonists of the p19 subunit of IL-23 to selectively inhibit IL-23 without disrupting the function of IL-12 cascades. $^{22,23}$ At this time, 3 inhibitors of the p19 subunit of IL-23 have been approved by the US Food and Drug Administration (FDA) and European Medicines Agency (EMA): guselkumab, tildrakizumab, and risankizumab. One other agent, mirikizumab, is undergoing Phase 3 of development.

\section{Guselkumab}

Guselkumab (Janssen Biotech, Inc., Horsham, PA, USA) is the first medication of its class approved by the FDA and EMA in 2017 for the treatment of adult patients with moderate-to-severe plaque psoriasis who are candidates for systemic therapy or phototherapy. ${ }^{17}$ Guselkumab is a fully human monoclonal antibody against the IL-23p19 subunit of IL-23 delivered as a $100 \mathrm{mg}$ subcutaneous injection dosed at weeks 0,4 , and then every 8 weeks. The p19 subunit is shared by both IL-23 and IL-39 cytokines. Blockade of the 19 subunit can in theory neutralize both IL-23 and IL-39, another pro-inflammatory cytokine. ${ }^{24}$ However, a role for IL-39 in the pathophysiology of psoriasis has not been established. ${ }^{24}$

\section{Treatment Efficacy}

The clinical efficacy of guselkumab in the treatment of moderate-to-severe psoriasis has been established in eight phase 3 and 4 clinical studies (Tables 1 and 2).

\section{VOYAGE Trials}

The VOYAGE trials were the earliest phase 3, randomized, double-blinded trials assessing the efficacy of guselkumab in patients with moderate-to-severe plaque psoriasis. $^{25,26}$ Subjects in the VOYAGE 1 were randomized into 3 groups: guselkumab $100 \mathrm{mg}$, placebo followed by guselkumab $100 \mathrm{mg}$, or adalimumab $80 \mathrm{mg}$ followed by adalimumab $40 \mathrm{mg}^{25}$ Co-primary endpoints were the proportions of patients who achieved clear or minimal disease as indicated by Investigator Global Assessment score (IGA 0/1) and who achieved a 90\% or greater improvement from baseline Psoriasis Area and Severity Index score (PASI 90). Secondary endpoints were measured by the scalp-specific IGA (ss-IGA), fingernail Physician Global Assessment (f-PGA), Nail Psoriasis Severity Index (NAPSI), Physician Global Assessment of hands and feet (hf-PGA), Dermatology Life Quality Index (DLQI), and Psoriasis Signs and Symptoms Diary (PSSD) scores.

Guselkumab was more effective than placebo as measured by IGA $0 / 1$ (85.1\% vs 6.9\%), PASI 90 (73.3\% vs $2.9 \%$ ), and all secondary endpoints at the end of week 16 (all $\mathrm{p}<0.001) .{ }^{25}$ Moreover, greater response to guselkumab compared to placebo was seen as early as 2 weeks. ${ }^{25}$ More subjects treated with guselkumab attained IGA $0 / 1$ than those treated with adalimumab $(80.5 \%$ vs $55.4 \%, \mathrm{p}<0.001)$ through week $48 .^{25}$ Similarly, PASI 90 was met by more patients in the guselkumab group $(76.3 \%)$ than those in the adalimumab group $(47.9 \%, \mathrm{p}<0.001)$ at week $48 .{ }^{25}$ While the proportions of patients meeting f-PGA $0 / 1$ (cleared/ minimal) were comparable between guselkumab and adalimumab groups at week 24, the proportion of patients attaining f-PGA 0/1 was higher in the guselkumab group at week $48(74.7 \%$ vs $61.8 \%, \mathrm{p}=0.038) .{ }^{25}$ However, the mean percent improvement in NAPSI scores was comparable between guselkumab and adalimumab at weeks 24 $(49.8 \%$ vs $49.4 \%)$ and $48 \quad(68.1 \%$ vs $61.4 \%){ }^{25}$ Guselkumab was more effective than adalimumab for all other secondary endpoints by week $48 .^{25}$

VOYAGE 2 evaluated the efficacy of guselkumab versus placebo and adalimumab, including one study arm with discontinuation of guselkumab and another arm that switched adalimumab non-responders to gulselkumab. ${ }^{26}$ Results from the placebo-controlled period (week 0-16) were comparable to that of VOYAGE $1 .{ }^{25,26}$ During the randomized withdrawal and re-treatment period (weeks 28-48), PASI 90 response was lost in the withdrawal group at a median of 23 weeks following the last guselkumab dose. ${ }^{26}$ Additionally, clinical responses (IGA, PASI) were greater in the guselkumab maintenance group than the withdrawal group through week $48(\mathrm{p}<0.001) .{ }^{26}$ Of the group of patients considered non-responders to 
Table I Phase 3 and 4 Clinical Trials

\begin{tabular}{|c|c|c|c|}
\hline Clinical Trial & $\begin{array}{l}\text { Number } \\
\text { of Patients }\end{array}$ & Study Design & Objective \\
\hline VOYAGE I (NCT0220723I) & 837 & $\begin{array}{l}\text { Phase } 3 \text {, multicenter, randomized, double-blind, } \\
\text { placebo- and active comparator-controlled trial. }\end{array}$ & $\begin{array}{l}\text { To compare efficacy and safety of guselkumab } \\
\text { with adalimumab and placebo in patients treated } \\
\text { for I year. }\end{array}$ \\
\hline VOYAGE 2 (NCT02207244) & 993 & $\begin{array}{l}\text { Phase } 3 \text {, multicenter, randomized, double blind, } \\
\text { placebo- and active comparator-controlled } \\
\text { study with a randomized withdrawal and } \\
\text { retreatment period. }\end{array}$ & $\begin{array}{l}\text { To assess efficacy and safety of guselkumab } \\
\text { versus placebo and adalimumab, including } \\
\text { interrupted treatment and switching } \\
\text { adalimumab nonresponders to guselkumab. }\end{array}$ \\
\hline NAVIGATE (NCT02203032) & 872 & $\begin{array}{l}\text { Phase 3, multicenter, randomized, double-blind } \\
\text { study. }\end{array}$ & $\begin{array}{l}\text { To evaluate the efficacy and safety of } \\
\text { guselkumab in patients with an inadequate } \\
\text { response to ustekinumab. }\end{array}$ \\
\hline ECLIPSE (NCT03090I00) & 1048 & $\begin{array}{l}\text { Phase 3, multicenter, double-blind, randomized, } \\
\text { comparator-controlled trial. }\end{array}$ & $\begin{array}{l}\text { To compare efficacy at week } 48 \text { for guselkumab } \\
\text { versus secukinumab. }\end{array}$ \\
\hline NCT02325219 & 192 & $\begin{array}{l}\text { Phase 3, multicenter, randomized, double-blind, } \\
\text { placebo-controlled study }\end{array}$ & $\begin{array}{l}\text { To evaluate efficacy and safety of guselkumab in } \\
\text { Japanese patients. }\end{array}$ \\
\hline ORION (NCT0290533I) & 78 & $\begin{array}{l}\text { Phase } 3 \text {, multicenter, double-blind, placebo- } \\
\text { controlled study. }\end{array}$ & $\begin{array}{l}\text { To evaluate the efficacy, safety, } \\
\text { pharmacokinetics, and acceptability of } \\
\text { guselkumab administered using a novel patient- } \\
\text { controlled injector (One-Press). }\end{array}$ \\
\hline IXORA-R (NCT03573323) & 1027 & $\begin{array}{l}\text { Phase 4, multicenter, randomized, double- } \\
\text { blinded, parallel-group study. }\end{array}$ & $\begin{array}{l}\text { To compare early and complete skin clearance } \\
\text { by ixekizumab versus guselkumab. }\end{array}$ \\
\hline POLARIS (NCT0295I533) & 119 & $\begin{array}{l}\text { Phase } 3 \mathrm{~b} \text {, multicenter, randomized, open-label, } \\
\text { assessor-blinded, active-comparator-controlled } \\
\text { study. }\end{array}$ & $\begin{array}{l}\text { To compare the efficacy and safety of } \\
\text { guselkumab with fumaric acid esters (FAE) in } \\
\text { patients with moderate-to-severe plaque } \\
\text { psoriasis who are naive to systemic treatment. }\end{array}$ \\
\hline
\end{tabular}

Note: Number of patients, study design and objective of clinical trials examining the use of guselkumab in the treatment of moderate-to-severe plaque psoriasis.

adalimumab and switched to guselkumab, $66.1 \%$ achieved PASI 90 at week 48 , and $28.6 \%$ achieved PASI $100 .{ }^{26}$ In long-term follow-up studies of the VOYAGE subjects, physician-reported (IGA and PASI) and patient-reported (DLQI and PSSD) outcomes were maintained through 3 and 4 years of continuous guselkumab treatment. ${ }^{27,28}$ Overall, guselkumab has greater efficacy with similar adverse events at a dosage of $100 \mathrm{mg}$ every 8 weeks compared to adalimumab.

VOYAGE 1 and 2 compared the clinical performance of guselkumab to adalimumab and both studies yielded similar results. ${ }^{25,26} \mathrm{In}$ a pooled analysis from VOYAGE 1 and VOYAGE 2, the response to guselkumab was similar in lighter and heavier patients while adalimumab was less effective for heavier patients than for lighter patients. ${ }^{29}$ Guselkumab was more effective than adalimumab and was also effective in patients who have failed adalimumab therapy.

\section{NAVIGATE}

NAVIGATE was a phase 3, randomized, double-blinded trial to evaluate the clinical efficacy of guselkumab in patients with moderate-to-severe psoriasis who did not adequately respond to ustekinumab, an IL-12/23 inhibitor. ${ }^{30}$ After initiating ustekinumab $(45 \mathrm{mg}$ or $90 \mathrm{mg}$, depending on weight), patients with IGA $\geq 2$ were randomized to receive guselkumab $100 \mathrm{mg}$ or to continue ustekinumab. Clinical response, measured by the number of visits patients achieved IGA $0 / 1$ and at least a relative 2-grade improvement, was higher in patients randomized to guselkumab compared to ustekinumab (1.5 vs 0.7 , $\mathrm{p}<0.001){ }^{30}$ The proportion of patients with PASI 90 response at week 28 was greater in the guselkumab group than ustekinumab group $(48.1 \%$ vs $22.6 \%$, $\mathrm{p}<0.001) .{ }^{30}$ A greater proportion of patients treated with guselkumab, compared to ustekinumab, achieved PASI 90 $(51.1 \%$ vs $24.1 \%, \mathrm{p}<0.001)$ and PASI $100(20.0 \%$ vs 
Table 2 Summary of Key Results of Clinical Trials

\begin{tabular}{|c|c|c|c|c|c|c|}
\hline \multirow[t]{2}{*}{ Clinical Trial } & & \multicolumn{5}{|c|}{ Proportion of Patients Achieving } \\
\hline & & IGA 0/I & DLQI 0/I & PASI 75 & PASI 90 & PASI 100 \\
\hline \multirow[t]{8}{*}{ VOYAGE I } & Week 16 & GUS $85.1 \%$ & GUS $56.3 \%$ & GUS $91.2 \%$ & GUS 73.3\% & GUS $37.4 \%$ \\
\hline & & ADM $65.9 \%$ & ADM $38.6 \%$ & ADM $73.1 \%$ & ADM $49.7 \%$ & ADM $17.1 \%$ \\
\hline & & PBO $6.9 \%$ & PBO $4.2 \%$ & PBO $5.7 \%$ & PBO $2.9 \%$ & PBO $0.6 \%$ \\
\hline & Week 24 & GUS $84.2 \%$ & GUS $60.9 \%$ & GUS $91.2 \%$ & GUS $80.2 \%$ & GUS $44.4 \%$ \\
\hline & & ADM $61.7 \%$ & ADM $39.5 \%$ & ADM $72.2 \%$ & ADM $53.0 \%$ & ADM $24.9 \%$ \\
\hline & Week 48 & GUS $80.5 \%$ & GUS $62.5 \%$ & GUS $87.8 \%$ & GUS $76.3 \%$ & GUS $47.4 \%$ \\
\hline & & ADM $55.4 \%$ & ADM $38.9 \%$ & ADM $62.6 \%$ & ADM $47.9 \%$ & ADM $23.4 \%$ \\
\hline & & All $p<0.00$ I & All $p<0.00$ I & All $p<0.00$ I & All $p<0.00$ I & All $p<0.00$ I \\
\hline \multirow[t]{6}{*}{ VOYAGE 2} & Week 16 & GUS $84.1 \%$ & GUS $51.7 \%$ & GUS $86.3 \%$ & GUS $70.0 \%$ & GUS 34.1\% \\
\hline & & ADM $67.7 \%$ & ADM $39.0 \%$ & ADM $68.5 \%$ & ADM $46.8 \%$ & ADM $20.6 \%$ \\
\hline & & PBO $8.5 \%$ & PBO 3.3\% & PBO 8.1\% & PBO2.4\% & PBO $0.8 \%$ \\
\hline & Week 24 & GUS $83.5 \%$ & GUS 57.6\% & GUS 89.1\% & GUS $75.2 \%$ & GUS $44.2 \%$ \\
\hline & & ADM $64.9 \%$ & ADM $41.1 \%$ & ADM $71.0 \%$ & ADM $54.8 \%$ & ADM $26.6 \%$ \\
\hline & & All $p<0.00$ I & All $p<0.00$ I & All $p<0.00$ I & All $p<0.00$ I & All $p<0.00$ I \\
\hline \multirow[t]{6}{*}{ NAVIGATE } & Week 28 & GUS 31.1\% & N/A & N/A & GUS $48.1 \%$ & $\mathrm{~N} / \mathrm{A}$ \\
\hline & & USM I4.3\% & & & USM $22.6 \%$ & \\
\hline & & $\mathrm{p}=0.00 \mathrm{I}$ & & & $p<0.001$ & \\
\hline & Week 52 & GUS $36.3 \%$ & GUS $38.8 \%$ & N/A & GUS $51.1 \%$ & GUS $20.0 \%$ \\
\hline & & USM 17.3\% & USM 19.0\% & & USM $24.1 \%$ & USM 7.5\% \\
\hline & & $\mathrm{p}<0.00 \mathrm{I}$ & & & $\mathrm{P}<0.001$ & $\mathrm{p}=0.003$ \\
\hline \multirow[t]{5}{*}{ ECLIPSE } & Week 12 & $N / A$ & N/A & GUS $89.3 \%$ & GUS $69.1 \%$ & $\mathrm{~N} / \mathrm{A}$ \\
\hline & & & & SKM $91.6 \%$ & SKM 76.1\% & \\
\hline & Week 48 & GUS $85.0 \%$ & N/A & N/A & GUS $84.5 \%$ & GUS $58.2 \%$ \\
\hline & & SKM $74.9 \%$ & & & SKM $70.0 \%$ & SKM 48.4\% \\
\hline & & & & & $\mathrm{p}<0.00 \mathrm{I}$ & \\
\hline NCT02325219 & Week 16 & GUS 50 mg 92.3\% & GUS 50 mg 64.1\% & GUS 50 mg 89.2\% & GUS 50 mg 70.8\% & GUS 50 mg 32.3\% \\
\hline & & GUS 100 mg $88.9 \%$ & GUS 100 mg 68.3\% & GUS 100 mg $84.1 \%$ & GUS $100 \mathrm{mg} 69.8 \%$ & GUS $100 \mathrm{mg} 27.0 \%$ \\
\hline & & PBO $7.8 \%$ & PBO 6.6\% & PBO $6.3 \%$ & PBO 0\% & PBO 0\% \\
\hline & & $\mathrm{p}<0.001$ & & $\mathrm{P}<0.00 \mathrm{I}$ & $\mathrm{p}<0.001$ & $\mathrm{p}<0.00 \mathrm{I}$ \\
\hline ORION & Week 16 & GUS $80.6 \%$ & $\mathrm{~N} / \mathrm{A}$ & GUS $88.7 \%$ & GUS $75.8 \%$ & GUS $50.0 \%$ \\
\hline & & PBO 0\% & & PBO 0\% & PBO 0\% & PBO 0\% \\
\hline & & $\mathrm{p}<0.001$ & & $\mathrm{P}<0.00 \mathrm{I}$ & $\mathrm{P}<0.00 \mathrm{I}$ & $\mathrm{p}<0.00 \mathrm{I}$ \\
\hline \multirow[t]{5}{*}{ IXORA-R } & Week 12 & N/A & $\mathrm{N} / \mathrm{A}$ & N/A & N/A & GUS $25 \%$ \\
\hline & & & & & & IXM 4I\% \\
\hline & & & & & & $p<0.001$ \\
\hline & Week 24 & $\mathrm{~N} / \mathrm{A}$ & $\mathrm{N} / \mathrm{A}$ & N/A & N/A & GUS $52 \%$ \\
\hline & & & & & & IXM 50\% \\
\hline \multirow[t]{3}{*}{ POLARIS } & Week 24 & N/A & GUS 62\% & GUS $90 \%$ & GUS $82 \%$ & GUS $32 \%$ \\
\hline & & & FAE I7\% & FAE $27 \%$ & FAE I4\% & FAE 3\% \\
\hline & & & $\mathrm{p}<0.001$ & $\mathrm{P}<0.00 \mathrm{I}$ & $\mathrm{p}<0.001$ & $\mathrm{P}<0.001$ \\
\hline
\end{tabular}

Note: All comparisons were made with guselkumab and $\mathrm{p}$-value represents significance of comparisons.

Abbreviations: IGA 0/I, Investigator Global Assessment score of 0 or I; PASI 75, at least a 75\% improvement in PASI score compared to baseline; PASI 90 , at least a $90 \%$ reduction in PASI score compared to baseline; PASI I00, at least a 100\% improvement in PASI score compared to baseline; DLQI 0/I, Dermatology Life Quality Index score of 0 or I; N/A, not available; GUS, guselkumab; ABM, adalimumab; PBO, placebo; USM, ustekinumab; SKM, secukinumab; IXM, ixekizumab, FAE, fumaric acid esters. 
$7.5 \%, \mathrm{p}<0.001)$ responses as well as the DLQI score of $0 / 1$ $(38.8 \%$ vs $19.0 \%)$ at week $52 .{ }^{30}$ This study identified guselkumab as a beneficial treatment option for psoriasis patients who did not respond to ustekinumab by week 16 .

\section{ECLIPSE}

ECLIPSE was a phase 3, randomized, double-blind headto-head trial that compared the efficacy and safety of guselkumab and secukinumab in patients with moderateto-severe plaque psoriasis. ${ }^{31}$ Secukinumab, an IL-17A inhibitor, is another approved treatment option for patients with psoriasis. Participants received either guselkumab $100 \mathrm{mg}$ and placebo injections to maintain the blind or secukinumab $300 \mathrm{mg}$ through week $44 .{ }^{31}$ Guselkumab was more effective than secukinumab in reaching the primary endpoint of PASI 90 ( $84 \%$ vs $70 \%, \mathrm{p}<0.001)$ at week $48 .^{31}$

ECLIPSE evaluated six major secondary endpoints in a fixed sequence to control for type 1 error. The first major secondary endpoint measured the proportions of patients in the guselkumab and secukinumab groups who achieved a PASI 75 response at both week 12 and $48 .^{31}$ As high as $84.6 \%$ of patients in the guselkumab group versus $80.2 \%$ of patients in the secukinumab group achieved a PASI 75 response both at week 12 and 48, which established noninferiority (margin of 10 percentage points) but not superiority. ${ }^{31}$ Therefore, statistical testing was not performed on subsequent major secondary endpoints. Patients in the guselkumab group, compared to the secukinumab group, achieved higher proportions of PASI 100 response $(58.2 \%$ vs $48.4 \%)$, IGA $0(62.2 \%$ vs $50.4 \%)$, and IGA $0 / 1(85.0 \%$ vs $74.9 \%)$ at week $48 .^{31}$

The major secondary endpoints measured at week 12 revealed a shift towards higher proportions of patients in the secukinumab group. As high as $89.3 \%$ of patients in the guselkumab group achieved PASI 75 at week 12 compared to $91.6 \%$ of patients in the secukinumab group. ${ }^{31}$ The PASI 100 response at week 12 in the guselkumab group was $69.1 \%$ versus $76.1 \%$ in the secukinumab group. ${ }^{31}$ ECLIPSE was a head-to-head trial showing the long-term efficacy of treatment with guselkumab is greater than that of secukinumab at week 48 .

\section{NCT02325219}

Ohtsuki and colleagues ${ }^{32}$ performed this phase 3 , randomized, double-blind, placebo-controlled study aiming to evaluate the efficacy of guselkumab in Japanese patients with moderate-to-severe plaque psoriasis. Patients were randomly assigned to receive guselkumab $50 \mathrm{mg}$ or
$100 \mathrm{mg}$ at weeks 0,4 , and then every 8 weeks or placebo with crossover to guselkumab $50 \mathrm{mg}$ or $100 \mathrm{mg}$ at week $16 .^{32}$ Co-primary endpoints were the portions of patients reaching IGA 0/1 and PASI 90 responses at week 16. At week 16, larger proportions of patients treated with guselkumab $50 \mathrm{mg}$ and $100 \mathrm{mg}$ versus placebo attained IGA 0/1 (92.3\% and $88.9 \%$ vs $7.8 \%, \mathrm{p}<0.001)$ and PASI $90(70.8 \%$ and $69.8 \%$ vs $0 \%, \mathrm{p}<0.001) .{ }^{32}$ More patients in the guselkumab $50 \mathrm{mg}$ and $100 \mathrm{mg}$ groups achieved PASI 75 response than did subjects receiving placebo $(89.2 \%$ and $84.1 \%$ vs $6.3 \%, \mathrm{p}<0.001$ ) at week $16 .{ }^{32}$ Guselkumab was more effective than placebo in the Japanese patient population in this study, consistent with findings in previous global studies.

\section{ORION}

ORION was a phase 3 , randomized, double-blind, placebocontrolled study assessing the efficacy of guselkumab, administered with a novel patient-controlled injector (One-Press), for moderate-to-severe psoriasis. Guselkumab has been previously studied using the UltraSafe Plus ${ }^{\mathrm{TM}}$ syringe which uses an automated delivery mechanism. ${ }^{25,26}$ One-Press allows the patient to manually control the injection speed rather than functioning as an autoinjector. ${ }^{33}$ Patients were randomized to receive guselkumab $100 \mathrm{mg}$ at weeks $0,4,12$, 20 , and 28 or placebo at weeks 0,4 , and 12 with crossover to guselkumab $100 \mathrm{mg}$ at weeks 16,20 , and $28 .{ }^{33}$ Co-primary endpoints were proportions of patients achieving IGA $0 / 1$ or PASI 90 response at week $16 .^{33}$

More patients in the guselkumab-treated group achieved IGA $0 / 1(80.6 \%$ vs $0 \%, \mathrm{p}<0.001)$ and PASI 90 $(75.8 \%$ vs $0 \%, \mathrm{p}<0.001)$ response than placebo-treated subjects at week $16 .{ }^{33}$ More patients treated with guselkumab achieved the major secondary endpoints of IGA 0 $(56.5 \%$ vs $0 \%, \mathrm{p}<0.001)$ and PASI $100(50.0 \%$ vs $0 \%$, $\mathrm{p}<0.001)$ responses, too. ${ }^{33}$ Ninety-nine percent of patients were satisfied or very satisfied with One-Press at week $28 .^{33}$ Steady-state serum concentrations were achieved by week 20 with the One-Press device, which is consistent with studies using the UltraSafe Plus syringe. ${ }^{33}$ This study demonstrated that administering guselkumab with the One-Press patient-controlled injector is efficacious and acceptable to patients with moderate-to-severe psoriasis.

\section{IXORA-R}

IXORA-R was a Phase 4, randomized, double-blind study comparing ixekizumab, an IL-17 inhibitor, to guselkumab in patients with moderate-to-severe psoriasis. $^{34}$ The 
primary objective was to compare early and complete skin clearance, measured by a primary endpoint of PASI 100 at week 12. Patients were randomized to either receive ixekizumab (160 mg starting dose, followed by $80 \mathrm{mg}$ every 2 weeks) or guselkumab (100 $\mathrm{mg}$ at weeks 0,4 , and 12). Placebo injections were given to patients in the guselkumab group to maintain blinding. At 12 weeks, the proportions of patients attaining PASI 100 for ixekizumab and guselkumab were $41 \%$ and $25 \%$, respectively $(\mathrm{p}<0.001){ }^{34}$ Ixekizumab was more effective than guselkumab at week 1 of treatment (median PASI improvement from baseline $34 \%$ vs $17 \%$, respectively). ${ }^{34}$ At week 24 , guselkmab and ixekizumab had similar PASI 100 responses ( $52 \%$ vs $50 \%$, $\mathrm{p}=0.41) .{ }^{24}$ PASI 100 responses were more rapid with ixekizumab than guselkumab by week 12 , but by week 24 guselkumab and ixekizumab were equally effective. ${ }^{24,34}$

\section{POLARIS}

Fumaric acid esters (FAE) are recommended in the European S3-Guidelines for the treatment of moderateto-severe plaque psoriasis and are commonly prescribed first-line treatment options in Germany. ${ }^{35,36}$ POLARIS was a phase 3, randomized, open-label, assessor-blinded, active-comparator-controlled trial evaluating the efficacy of guselkumab with that of FAEs in patients with moderate-to-severe plaque psoriasis naïve to systemic treatment. ${ }^{37}$ Subjects were randomized to receive either daily oral FAEs as a fixed mixture of dimethyl fumarate (induction with $30 \mathrm{mg}$ and maintenance/tapering with $120 \mathrm{mg}$ ), or guselkumab (100 $\mathrm{mg}$ at week 0,4 , and then every 8 weeks). At week 24, guselkumab was more effective than FAE as measured by PASI 75 (90\% vs $27 \%$ ), PASI 90 ( $82 \%$ vs $14 \%$ ), PASI 100 (32\% vs $3 \%$ ), and DLQI scores of $0 / 1$ (62\% vs $17 \%)$, all $\mathrm{p}<0.001 .{ }^{37}$ Additionally, guselkumab, achieving PASI 90 as early as 4 weeks, had faster onset of efficacy compared to FAE.$^{37}$ Overall, FAE was less effective than guselkumab for treating moderate-to -severe psoriasis.

\section{Safety}

Several clinical trials validated the consistent safety profile of guselkumab. A Phase 1 study (NCT01484587) reported pruritus, folliculitis, nasopharyngitis, and injection-site erythema as the most common adverse events (AEs). ${ }^{38}$ A Phase 2 study similarly reported infections as the most common AEs but also reported a case of cancer (grade 3 cervical intraepithelial neoplasia) and 3 major adverse cardiovascular events among patients receiving guselkumab. ${ }^{39}$ Neither phase 1 nor 2 studies found evidence of dose-related AEs. Multiple phase 2 and 3 studies revealed similar rates of AEs and serious AEs in both guselkumab and placebo groups. ${ }^{25,26,39}$ The pivotal VOYAGE trials reported the most common AEs as nasopharyngitis, headache, and upper respiratory tract infections. Injection site reactions were mild and uncommon. As high as $6.6 \%$ and $9.0 \%$ of patients had positive antibodies to guselkumab at week 48 and 60 , respectively. ${ }^{25,26,34}$ However, the immunogenicity of guselkumab was not clinically relevant as no association exists between efficacy and development of anti-drug antibodies or adverse events. ${ }^{40}$ No Crohn's disease, anaphylactic, or serum sickness-like reactions were reported in any of the identified clinical trials. Moreover, rates of AEs did not increase over three years of continuous treatment with guselkumab. ${ }^{27}$ These safety findings were maintained even after four years of continuous guselkumab treatment. $^{28}$

There are no published results regarding the safety of guselkumab in patients who are pregnant and/or breastfeeding. ${ }^{41}$ Additionally, there are no age-related differences in drug clearance in patients $\geq 65$ years old compared to those $<65$ years old. Therefore, there is no need to adjust dose based on age. ${ }^{41}$ Although there are weightrelated differences in clearance and volume of distribution, no studies have published safety reasons to dose guselkumab based on weight. ${ }^{29,41}$

Phase 3 trials revealed comparable rates of AEs between guselkumab and other biologics (adalimumab, secukinumab, ixekizumab). ${ }^{25,26,31,34}$ However, the NAVIGATE study revealed a slightly higher incidence of adverse events in the guselkumab (64.4\%) group compared to ustekinumab (55.6\%). These AEs were mostly infections (nasopharyngitis) and musculoskeletal complaints (back pain, psoriatic arthritis). ${ }^{30}$ Fewer patients discontinued guselkumab than FAE treatment due to AEs $(0.0 \%$ vs $28.0 \%, \mathrm{p}<0.001) .{ }^{37}$ Guselkumab is a welltolerated and safe treatment option for psoriasis.

\section{Discussion}

The ability to selectively target the IL-23/Th17 axis has shifted the paradigm of the management of psoriasis. Several highly efficacious systemic therapies target this pathway with excellent safety profiles. This is demonstrated by the clinical efficacy of IL-23p19 inhibitors and IL-17 inhibitors for the treatment of moderate-to- 
severe psoriasis. IL-17 inhibitors were more effective than TNF- $\alpha$ inhibitors etanercept and adalimumab and the IL-12/23 inhibitor ustekinumab for the treatment of moderate-to-severe psoriasis in head-to-head clinical trials. ${ }^{14}$ However, adverse effects associated with IL-17 inhibitors such as mucocutaneous Candida infections and triggering or worsening of inflammatory bowel disease, in addition to the pursuit of increasingly effective medications, created a need for alternative therapies targeting this pathway.

With the discovery of IL-23 as the key regulator of Th17 cells, several antagonists of the p19 subunit of IL-23 have been tested and approved for the treatment of psoriasis, including guselkumab, tildrakizumab, and risankizumab. Mirikizumab, another agent targeting IL-23p19, recently completed phase 3 of clinical trial investigations with positive results and is undergoing submission to regulatory authorities for approval. ${ }^{42}$ In contrast to ustekinumab, this medication class allows IL-12-dependent functions to remain intact, preserving the IL-12/Th1 axis vital in the innate and adaptive immune defense against intracellular pathogens and malignant cells. ${ }^{43-45}$ The IL-12 cytokine may also have an anti-inflammatory effect on Th17-centered inflammation in the skin by promoting the differentiation of Th17 cells into regulatory $\mathrm{T}$ cell or Th1 cell populations. ${ }^{14,16}$

Guselkumab is highly efficacious and safe in treating moderate-to-severe psoriasis. In head-to-head trials guselkumab was more effective than adalimumab, ustekinumab, secukinumab, and fumaric acid esters. Analysis of response-over-time curves in ECLIPSE reveals that secukinumab achieved a faster onset of response through week 16 , but after week 20 the efficacy favored guselkumab which was maintained through one year. ${ }^{46}$ Ixekizumab, another anti-IL-17 antibody, was faster acting than guselkumab in IXORA-R, but the two drugs were equally effective at week 24; relative efficacy at longer times was not evaluated. $^{24}$

Efficacy and speed of improvement are important parameters when selecting treatment options, especially when a drug that provides the fastest improvement does not exhibit the highest long-term efficacy. Dosing regimens vary widely among available biologic therapies. Guselkumab has a less frequent dosing regimen (every 8 weeks) when compared to anti-IL-17 agents (every 2-4 weeks) which can contribute to increased therapy adherence and disease control. A patient-centered, individual approach to medication selection that incorporates a discussion of parameters such as efficacy, speed of improvement, and dosing regimens would increase the likelihood of achieving treatment goals for patients with moderate-to-severe psoriasis.

\section{Conclusion}

Guselkumab is a monoclonal antibody selectively targeting IL-23p19 and the first in its class approved to treat moderate-to-severe psoriasis. This class of medication is quickly expanding to comprise a large section of the biologics market for psoriasis with the approval of tildrakizumab and risankizumab and the likely approval of mirikizumab in the future. The excellent efficacy and safety profiles of guselkumab continue to be supported by recent studies displaying great potential in long-term treatment of psoriasis. The effective and safe profile, convenient dosing, and improved quality of life in patients make gulselkumab a viable first-line treatment option for moderate-to-severe psoriasis. ${ }^{47}$

\section{Funding}

There is no funding to report.

\section{Disclosures}

Feldman received research, speaking and/or consulting support from Galderma, GSK/Stiefel, Almirall, Alvotech, Leo Pharma, BMS, Boehringer Ingelheim, Mylan, Celgene, Pfizer, Ortho Dermatology, Abbvie, Samsung, Janssen, Lilly, Menlo, Helsinn, Arena, Forte, Merck, Novartis, Regeneron, Sanofi, Novan, Qurient, National Biological Corporation, Caremark, Advance Medical, Sun Pharma, Suncare Research, Informa, UpToDate and National Psoriasis Foundation. He consults for others through Guidepoint Global, Gerson Lehrman and other consulting organizations. $\mathrm{He}$ is founder and majority owner of www.DrScore.com. He is also a founder and part owner of Causa Research, a company dedicated to enhancing patients' adherence to treatment. Jeremy Light and Jennifer $\mathrm{Su}$ have no conflicts to disclose.

\section{References}

1. Mehta NN, Azfar RS, Shin DB, Neimann AL, Troxel AB, Gelfand JM. Patients with severe psoriasis are at increased risk of cardiovascular mortality: cohort study using the general practice research database. Eur Heart J. 2009;31(8):1000-1006. doi:10.1093/eurheartj/ehp567

2. Jindal S, Jindal N. Psoriasis and cardiovascular diseases: a literature review to determine the causal relationship. Cureus. 2018;10(2):e2195. doi:10.7759/cureus.2195 
3. Fu Y, Lee C-H, Chi -C-C. Association of psoriasis with inflammatory bowel disease. JAMA Dermatol. 2018;154(12):1417. doi:10.1001/ jamadermatol.2018.3631

4. Kurd SK, Troxel AB, Crits-Christoph P, Gelfand JM. The risk of depression, anxiety and suicidality in patients with psoriasis: a population-based cohort study. Arch Dermatol. 2010;146 (8):891-895. doi:10.1001/archdermatol.2010.186

5. Koo J, Marangell LB, Nakamura M, et al. Depression and suicidality in psoriasis: review of the literature including the cytokine theory of depression. J Eur Acad Dermatol Venereol. 2017;31(12):1999-2009. doi:10.1111/jdv.14460

6. Bhutani T, Patel T, Koo B, Nguyen T, Hong J, Koo J. A prospective, interventional assessment of psoriasis quality of life using a nonskin-specific validated instrument that allows comparison with other major medical conditions. J Am Acad Dermatol. 2013;69(2): e79-e88. doi:10.1016/j.jaad.2012.10.009

7. Gelfand JM. Commentary: does biologic treatment of psoriasis lower the risk of cardiovascular events and mortality? J Am Acad Dermatol. 2018;79(1):69-70. doi:10.1016/j.jaad.2018.03.046

8. Wu JJ, Sundaram M, Cloutier M, et al. The risk of cardiovascular events in psoriasis patients treated with tumor necrosis factor- $\alpha$ inhibitors versus phototherapy: an observational cohort study. $\mathrm{J} \mathrm{Am}$ Acad Dermatol. 2018;79(1):60-68. doi:10.1016/j.jaad.2018.02.050

9. Mehta NN, Shin DB, Joshi AA, et al. Effect of 2 psoriasis treatments on vascular inflammation and novel inflammatory cardiovascular biomarkers. Circ Cardiovasc Imaging. 2018;11(6). doi:10.1161/ circimaging. 117.007394

10. Sulzberger MB, Witten VH. The effect of topically applied compound $\mathrm{f}$ in selected dermatoses. $J$ Invest Dermatol. 1952;19 (2):101-102. doi:10.1038/jid.1952.72

11. Meuller W, Hermann B. Cyclosporin a for psoriasis. $N$ Engl J Med. 1979;301(10):555. doi:10.1056/nejm197909063011015

12. Winterfield LS. Psoriasis treatment: current and emerging directed therapies. Ann Rheum Dis. 2005;64(suppl_2):ii87-ii90. doi:10.1136/ ard.2004.032276

13. Torres T. Selective interleukin-23 p19 inhibition: another game changer in psoriasis? focus on risankizumab. Drugs. 2017;77 (14):1493-1503. doi:10.1007/s40265-017-0794-1

14. Hawkes JE, Yan BY, Chan TC, Krueger JG. Discovery of the IL-23/ IL-17 signaling pathway and the treatment of psoriasis. J Immunol. 2018;201(6):1605-1613. doi:10.4049/jimmunol.1800013

15. Chan TC, Hawkes JE, Krueger JG. Interleukin 23 in the skin: role in psoriasis pathogenesis and selective interleukin 23 blockade as treatment. Ther Adv Chronic Dis. 2018;9(5):111-119. doi:10.1177/ 2040622318759282

16. Hawkes JE, Chan TC, Krueger JG. Psoriasis pathogenesis and the development of novel targeted immune therapies. J Allergy Clin Immunol. 2017;140(3):645-653. doi:10.1016/j.jaci.2017.07.004

17. Fragoulis GE, Siebert S, McInnes IB. Therapeutic targeting of IL-17 and IL-23 cytokines in immune-mediated diseases. Annu Rev Med. 2016;67(1):337-353. doi:10.1146/annurev-med-051914-021944

18. Piskin G, Sylva-Steenland RMR, Bos JD, Teunissen MBM. In vitro and in situ expression of IL-23 by keratinocytes in healthy skin and psoriasis lesions: enhanced expression in psoriatic skin. J Immunol. 2006;176(3):1908-1915. doi:10.4049/jimmunol.176.3.1908

19. Lee E, Trepicchio WL, Oestreicher JL, et al. Increased expression of interleukin 23 p19 and p40 in lesional skin of patients with psoriasis vulgaris. J Exp Med. 2004;199(1):125-130. doi:10.1084/jem.20030451

20. Nestle FO, Kaplan DH, Barker J. Psoriasis. N Engl J Med. 2009;361 (5):496-509. doi:10.1056/NEJMra0804595

21. Di Cesare A, Di Meglio P, Nestle FO. The IL-23/Th17 axis in the immunopathogenesis of psoriasis. J Invest Dermatol. 2009;129 (6):1339-1350. doi:10.1038/jid.2009.59

22. Machado Á, Torres T. Guselkumab for the treatment of psoriasis. BioDrugs. 2018;32(2):119-128. doi:10.1007/s40259-018-0265-6
23. Yang EJ, Smith MP, Ly K, Bhutani T. Evaluating guselkumab: an anti-IL-23 antibody for the treatment of plaque psoriasis. Drug Des Devel Ther. 2019;13:1993-2000. doi:10.2147/dddt.s137588

24. Blauvelt A, Leonardi C, Elewski B, et al. A head-to-head comparison of ixekizumab versus guselkumab in patients with moderate-to-severe plaque psoriasis: 24-week efficacy and safety results from a randomised, doubleblinded trial. Br J Dermatol. 2020;182(6):1348-1358. doi:10.1111/bjd.19509

25. Blauvelt A, Papp KA, Griffiths CEM, et al. Efficacy and safety of guselkumab, an anti-interleukin-23 monoclonal antibody, compared with adalimumab for the continuous treatment of patients with moderate to severe psoriasis: results from the Phase III, double-blinded, placebo- and active comparator-controlled VOYAGE 1 trial. $\mathrm{J} \mathrm{Am}$ Acad Dermatol. 2017;76(3):405-417. doi:10.1016/j.jaad.2016.11.041

26. Reich K, Armstrong AW, Foley P, et al. Efficacy and safety of guselkumab, an anti-interleukin-23 monoclonal antibody, compared with adalimumab for the treatment of patients with moderate to severe psoriasis with randomized withdrawal and retreatment: results from the phase III, double-blind, placebo- and active comparatorcontrolled VOYAGE 2 trial. J Am Acad Dermatol. 2017;76 (3):418-431. doi:10.1016/j.jaad.2016.11.042

27. Reich K, Griffiths CEM, Gordon KB, et al. Maintenance of clinical response and consistent safety profile with up to 3 years of continuous treatment with guselkumab: results from the VOYAGE 1 and VOYAGE 2 trials. $\mathrm{J} \mathrm{Am} \mathrm{Acad}$ Dermatol. 2020;82(4):936-945. doi:10.1016/j.jaad.2019.11.040

28. Reich K, Armstrong AW, Foley P, et al. Maintenance of response through up to 4 years of continuous guselkumab treatment of psoriasis in the VOYAGE 2 phase 3 study. Am J Clin Dermatol. 2020;21 (6):881-890. doi:10.1007/s40257-020-00555-7

29. Gordon KB, Blauvelt A, Foley P, et al. Efficacy of guselkumab in subpopulations of patients with moderate-to-severe plaque psoriasis: a pooled analysis of the Phase III VOYAGE 1 and VOYAGE 2 studies. Br J Dermatol. 2017;178(1):132-139. doi:10.1111/bjd.16008

30. Langley RG. Efficacy and safety of guselkumab in patients with psoriasis who have an inadequate response to ustekinumab: results of the randomized, double-blind, phase III NAVIGATE trial. $\mathrm{Br}$ J Dermatol. 2017;178(1):114-123. doi:10.1111/bjd.15750

31. Reich K, Armstrong AW, Langley RG, et al. Guselkumab versus secukinumab for the treatment of moderate-to-severe psoriasis (ECLIPSE): results from a phase 3, randomised controlled trial. Lancet. 2019;394 (10201):831-839. doi:10.1016/s0140-6736(19)31773-8

32. Ohtsuki M, Kubo H, Morishima H, Goto R, Zheng R, Nakagawa H. Guselkumab, an anti-interleukin-23 monoclonal antibody, for the treatment of moderate to severe plaque-type psoriasis in Japanese patients: efficacy and safety results from a phase 3, randomized, double-blind, placebo-controlled study. $J$ Dermatol. 2018;45 (9):1053-1062. doi:10.1111/1346-8138.14504

33. Ferris LK, Ott E, Jiang J, et al. Efficacy and safety of guselkumab, administered with a novel patient-controlled injector (one-press), for moderate-tosevere psoriasis: results from the phase 3 ORION study. J Dermatol Treat. 2019;31(2):152-159. doi:10.1080/09546634.2019.1587145

34. Blauvelt A, Papp K, Gottlieb A, et al. A head-to-head comparison of ixekizumab vs. guselkumab in patients with moderate-to-severe plaque psoriasis: 12-week efficacy, safety and speed of response from a randomized, double-blinded trial. Br J Dermatol. 2020;182 (6):1348-1358. doi:10.1111/bjd.18851

35. Balak DMW. Fumaric acid esters in the management of psoriasis. Psoriasis. 2015;5:9-23. doi:10.2147/ptt.s51490

36. Nast A, Spuls PI, van der Kraaij G, et al. European S3-guideline on the systemic treatment of psoriasis vulgaris - update apremilast and secukinumab - EDF in cooperation with EADV and IPC. J Eur Acad Dermatol Venereol. 2017;31(12):1951-1963. doi:10.1111/jdv.14454

37. Thaçi D, Pinter A, Sebastian M, et al. Guselkumab is superior to fumaric acid esters in patients with moderate-to-severe plaque psoriasis who are naive to systemic treatment: results from a randomized, active-comparator-controlled phase IIIb trial (POLARIS). $\mathrm{Br}$ J Dermatol. 2020;183(2):265-275. doi:10.1111/bjd.18696 
38. Nemoto O, Hirose K, Shibata S, Li K, Kubo H. Safety and efficacy of guselkumab in Japanese patients with moderate-to-severe plaque psoriasis: a randomized, placebo-controlled, ascending-dose study. Br J Dermatol. 2018;178(3):689-696. doi:10.1111/bjd.16236

39. Gordon KB, Duffin KC, Bissonnette R, et al. A phase 2 trial of guselkumab versus adalimumab for plaque psoriasis. $N$ Engl J Med. 2015;373(2):136-144. doi:10.1056/NEJMoa1501646

40. Zhu Y, Marini JC, Song M, et al. Immunogenicity of guselkumab is not clinically relevant in patients with moderate-to-severe plaque psoriasis. J Invest Dermatol. 2019;139(8):1830-1834.e6. doi:10.10 16/j.jid.2019.02.018

41. Galluzzo M, D’Adamio S, Campione E, Bianchi L, Talamonti M. A safety evaluation of guselkumab for the treatment of psoriasis. Expert Opin Drug Saf. 2018;17(7):741-751. doi:10.1080/14740 338.2018.1488963

42. Eli Lilly and Company. Lilly's Mirikizumab Superior to Cosentyx (Secukinumab) in a Phase 3 Study for Patients with Moderate to Severe Plaque Psoriasis. Eli Lilly and Company; 2020.
43. Liu J, Cao S, Kim S, et al. Interleukin-12: an update on its immunological activities, signaling and regulation of gene expression. Curr Immunol Rev. 2005;1(2):119-137. doi:10.2174/1573395054065115

44. Kleinschek MA, Muller U, Brodie SJ, et al. IL-23 enhances the inflammatory cell response in cryptococcus neoformans infection and induces a cytokine pattern distinct from IL-12. J Immunol. 2006;176(2):1098-1106. doi:10.4049/jimmunol.176.2.1098

45. Langowski JL, Zhang X, Wu L, et al. IL-23 promotes tumour incidence and growth. Nature. 2006;442(7101):461-465. doi:10.1038/ nature 04808

46. Light JG, Haidari W, Feldman SR. Assessing efficacy and the speed of response in psoriasis treatment. J Dermatol Treat. 2019;30 (6):523-524. doi:10.1080/09546634.2019.1643588

47. Mrowietz U, Kragballe K, Reich K, et al. Definition of treatment goals for moderate to severe psoriasis: a European consensus. Arch Dermatol Res. 2010;303(1):1-10. doi:10.1007/s00403-010-1080-1

\section{Publish your work in this journal}

Clinical, Cosmetic and Investigational Dermatology is an international, peer-reviewed, open access, online journal that focuses on the latest clinical and experimental research in all aspects of skin disease and cosmetic interventions. This journal is indexed on CAS
The manuscript management system is completely online and includes a very quick and fair peer-review system, which is all easy to use. Visit http://www.dovepress.com/testimonials.php to read real quotes from published authors. 